\title{
Causas De ausentismo En El Programa De Becas De Capacitación De Fundación Trauma En Argentina
}

\author{
Carlos Tenaillon ${ }^{1}$, Laura Bosque ${ }^{2}$, Ezequiel Monteverde ${ }^{3}$, Joaquín Baliña ${ }^{4}$, Jorge Neira ${ }^{5}$, Laura Ruffa ${ }^{6}$
}

\begin{abstract}
Introduction and objectives: The trauma foundation (FT) promotes training, with scientific support through a Scholarship Program. From 2009 to 2015 , TF awarded 7,021 scholarships to 3,944 professionals (1.8 scholarship/person with an average of 1000 scholarships/year), registering an absenteeism (nonattendance) of $18.2 \%$. The objective of the present work was to analyze the causes of absenteeism and to implement strategies to reduce it.

Methods: An online survey was prepared with 12 multiple choice questions, which was sent to the 756 absentees who had email (the total number of absentees was 1,184), of which $168(22 \%)$ responded.

Results: Of the total survey responses, $66.7 \%$ were women and most of the respondents were in the age range of $31-40$ years (34.5\%). $51.8 \%$ were doctors, and $44 \%$ reported having a formal employment relationship. The causes of absenteeism: $39.9 \%$ due to challenges at work; $14.9 \%$ for personal reasons; $3 \%$ due to lack of time to study; $3 \%$ due to lack of interest in the course; $2.4 \%$ due to difficulties in accessing/downloading the reading material and $36.9 \%$ did not specify. $15.5 \%$ responded that they had been absent in more than one course, $15.5 \%$ that they did not remember which course they were absent and $12.5 \%$ mentioned $\mathrm{ACLS} \mathrm{S}^{\odot}$.

Conclusion: The results of the survey allowed us to create strategies to reduce absenteeism. Since the main cause of absenteeism was workrelated issues, it is necessary to negotiate with the health authorities to provide protected time to the scholars to leave work and attend the courses (through replacements, permissions or flexibility in work hours). Despite the Scholarship Program eliminating one economic barrier (training costs are paid for), we observed that it is not enough to guarantee access to the courses. This finding does not eliminate the importance of promoting individual commitment of the scholars to attend the training and reduce absenteeism, with the aim of improving the performance of their role in the healthcare field.
\end{abstract}

Keywords: Absenteeism, Argentina, Grant, Improvement, Program, Quality, Registry, Results, Training, Trauma.

Panamerican Journal of Trauma, Critical Care \& Emergency Surgery (2019): 10.5005/jp-journals-10030-1246

\section{Resumen}

Introducción y objetivos: Fundación Trauma (FT) promueve la capacitación con respaldo científico a través de un Programa de Becas que entre 2009 y 2015 entregó 7021 vacantes a 3944 profesionales (1,8 beca/persona con un promedio de 1000 becas/año), registrando un ausentismo de 18,2\%. El objetivo de este trabajo fue analizar las causas de ausentismo para implementar estrategias de mejora.

Métodos: Se elaboró una encuesta online con 12 preguntas multiple choice, que se envió a los 756 ausentes que contaban con email (el ausentismo total fue 1184), de los cuales respondieron 168 (22\%).

Resultados: Del total de encuestas respondidas el 66,7\% fue de mujeres, el rango etario de mayor proporción de réplicas fue 31-40 años (34,5\%). El 51,8\% fueron médicos, y el 44\% refirió tener relación laboral formal. Causas de ausentismo: 39,9\% por dificultades laborales; 14,9\% por cuestiones personales; $3 \%$ por falta de tiempo para estudiar; $3 \%$ por falta de interés en el curso; $2,4 \%$ por dificultades para acceder/descargar el material de lectura y $36,9 \%$ no especificó. El 15,5\% manifestó haber estado ausente en más de un curso, 15,5\% refirió no recordar en qué curso estuvo ausente y el $12,5 \%$ mencionó el $\mathrm{ACLS}^{\circledast}$.

Conclusión: Los resultados de la encuesta permiten orientar las estrategias para disminuir el ausentismo. Debido a que la causa principal de ausentismo fueron motivos laborales, es necesario trabajar con las autoridades sanitarias para garantizar la asistencia de los becados (mediante reemplazos, autorizaciones o flexibilidad horaria). Si bien el Programa de Becas elimina la barrera económica, se observa que no es suficiente para garantizar el acceso a los cursos. Esto no soslaya la importancia de fomentar el compromiso individual de los becados para reducir el ausentismo con el objetivo de mejorar el desempeño de su rol en el ámbito asistencial.

Keywords: Argentina, Ausentismo, Beca, Calidad, Capacitación, Mejora, Programa, Registro, Resultado, Trauma.

\section{Introducción y OBjetivos}

En el año 2009 Fundación Trauma (FT), una organización no gubernamental sin fines de lucro, inició un Programa de Becas en cursos con respaldo científico sin precedentes en Argentina, con el objetivo de promover la capacitación y actualización de los profesionales vinculados a la atención de pacientes traumatizados. El mismo se implementó en instituciones que participan de la Red de Trauma del Ministerio de Salud de la Provincia de Buenos Aires, Argentina.

La experiencia de los países que han desarrollado sistemas de trauma demuestra que el nivel de categorización de un centro
${ }^{1-6}$ Department of Capacitacion, Fundacion Trauma, Buenos Aires, Argentina

Corresponding Author: Carlos Tenaillon, Department of Capacitacion, Fundacion Trauma, Buenos Aires, Argentina, Phone: +54 911 41936623, e-mail: ctenaillon@fundaciontrauma.org.ar

How to cite this article: Tenaillon C, Bosque L, et al. Causas De ausentismo En El Programa De Becas De Capacitación De Fundación Trauma En Argentina. Panam J Trauma Crit Care Emerg Surg 2019;8(2):75-79.

Source of support: Registro de Capacitación

Conflict of interest: None

oThe Author(s). 2019 Open Access This article is distributed under the terms of the Creative Commons Attribution 4.0 International License (https://creativecommons. org/licenses/by-nc/4.0/), which permits unrestricted use, distribution, and non-commercial reproduction in any medium, provided you give appropriate credit to the original author(s) and the source, provide a link to the Creative Commons license, and indicate if changes were made. The Creative Commons Public Domain Dedication waiver (http://creativecommons.org/publicdomain/zero/1.0/) applies to the data made available in this article, unless otherwise stated. 
Tabla 1: Cursos por sociedad cientifica

\begin{tabular}{|c|c|}
\hline Sociedad científica & Cursos \\
\hline Asociación Argentina de Cirugia (AAC) & Advanced Trauma Life Support (ATLS ${ }^{\circledast}$ ) \\
\hline Asociación Argentina de Quemaduras (AAQ) & Atención Básica Inicial de Quemados (ABIQ $\left.{ }^{\circledR}\right)$ \\
\hline Fundación Cardiológica Arge ntina (FCA) & Advanced Cardiac Life Support (ACLS $\left.{ }^{\circledast}\right)$ \\
\hline \multirow[t]{6}{*}{ Sociedad Argentina de Medicina y Cirugía del Trauma (SAMCT) } & Advanced Disaster Medical Response (ADMR $\left.{ }^{\oplus}\right)$ \\
\hline & Advanced Trauma Care for Nurses (A TCN $\left.{ }^{\circledR}\right)$ \\
\hline & Curso de Socorrismo Avanzado (CSA $)^{\oplus}$ \\
\hline & Curso de Socorrismo Intennedio $\left(\mathrm{CSI}^{\oplus}\right)$ \\
\hline & Curso de Socorrismo Pediátrico (CSP®) \\
\hline & Ultrasonografia en Emergencias y $\left.\operatorname{Trauma}_{(\mathrm{USET}}{ }^{\oplus}\right)$ \\
\hline \multirow[t]{3}{*}{ Sociedad Argentina de Pediatría (SAP) } & Pediatric Advanced Life Support (PALS $\left.{ }^{\circledast}\right)$ \\
\hline & Reanimación Avanzada Pediátrica para Enfermeros (RAPEnf.) \\
\hline & Reanimación Avanzada Pediátrica para Médicos (RAPMéd.) \\
\hline \multirow[t]{5}{*}{ Sociedad Argentina de Terapia Intensiva (SA TI) } & Curse de Ventilación Mecánica en Adultos (CVM-A $\left.{ }^{\oplus}\right)$ \\
\hline & Curse de Ventilación Mecánica Pediatrica (CVM-P®) \\
\hline & Enfermeria en Cuidados Crlticos (ECC $\left.{ }^{\circledast}\right)$ \\
\hline & Fundamental Critical Care Support (FCCS $\left.{ }^{\circledR}\right)$ \\
\hline & Pediatric Fundamental Critical Care Support (PFCCS $\left.{ }^{\circledast}\right)$ \\
\hline
\end{tabular}

depende de distintos requerimientos, dentro de los cuales se encuentra la formación del recurso humano.

FT se basa para armar la oferta de becas en las recomendaciones de la CICCATED $^{1}$ que definió los cursos que son esenciales y deseables de acuerdo al rol que desempeñan los profesionales en el proceso de atención.

Para ello, FT firmó convenios con las sociedades científicas (SC) que ofrecen los cursos seleccionados (Tabla 1).

Independientemente de que las oportunidades de asistir a los cursos se ofrecen en forma totalmente gratuita a los participantes, el grado de ausentismo registrado es alto, con una proporción general de 18,2\% (IC95\% 17.3-19.1). Con el objetivo de identificar estos factores y evaluar la posibilidad de estrategias de mejora, se llevó a cabo este estudio en una muestra de profesionales de la salud que recibieron alguna beca por parte del programa de FT.

\section{Métodos}

FT desarrolló un Sistema de Registro que incluye registro de trauma (RT), registro de capacitación (RC), módulo de reportes y módulos para la comunicación inter e intra-institucional y la administración de usuarios. La sección de comunicación, llamada Comunidad Trauma ofrece acceso a novedades de interés para la especialidad, noticias sobre oferta de cursos y a la promoción de actividades realizadas por instituciones nacionales e internacionales afines al trauma. El RT tiene versiones destinadas al hospital y al ámbito prehospitalario. Ambas aplicaciones ofrecen acceso a diferentes secciones dependiendo del perfil del usuario. EI RC es una herramienta que permite realizar un relevamiento del perfil de capacitación de los profesionales de cada institución. Esta información incluye datos personales, profesionales y de capacitación previa. Los datos que se obtienen del análisis de esta aplicación son de gran importancia para administrar el Programa de Becas. El primer paso para acceder al este programa es el registro en la aplicación, iniciado por el profesional al acceder a la plataforma on-line, donde ingresa sus datos personales y de la institución en la cual se desempeña. A partir de ese momento, el responsable del programa (RP) valida la información básica y el profesional pasa a la segunda etapa de relevamiento de información detallada (ocupación, especialidad, área o servicio, días de trabajo y capacitación previa, ingresando fechas y cursos específicos).

Luego del relevamiento detallado, el profesional puede acceder al Programa de Becas ingresando al RC y seleccionando el curso de interés en el calendario de fechas. El pedido de beca llega mediante un aviso automático al área de Capacitación de FT, donde se evalúa la pertinencia de acuerdo con el perfil y rol del profesional, y comunica la solicitud al RP en la institución de dicha solicitud para su aprobación o rechazo. Junto a la solicitud, se adjunta una reseña del perfil profesional y sus antecedentes dentro del Programa de Becas.

FT promueve que la aprobación o rechazo de becas sea un proceso participativo que incluya al RP, a jefes de servicios, a los integrantes de los comités de Trauma y de Docencia e Investigación. FT no solicita ninguna contraprestación por el otorgamiento de las becas a las instituciones o sus profesionales, para lo cual cuenta con financiación propia.

Mediante los convenios firmados con las SC, FT busca garantizar un calendario acorde a las necesidades del Programa, se acuerdan los aranceles y se establece la modalidad de inscripción y posterior envío de resultados.

FT promueve que las capacitaciones sean realizadas dentro de cada institución, siempre que estas cumplan con los requisitos para su dictado, evitando así que los profesionales deban trasladarse.

Para los fines de este trabajo, realizamos una encuesta on-line anónima y de participación voluntaria utilizando un formulario de Google. La misma fue diseñada para registrar ausentismo en un máximo de tres cursos, por lo que contó con un mínimo de 8 y un máximo de 12 preguntas de opción múltiple (ver Anexo I). Los profesionales contaron con 67 días para responder la encuesta desde el momento de haberla recibido.

\footnotetext{
${ }^{1}$ Coalición Intersocietaria para la Certificación Profesional y la Categorización y Acreditación Institucional en Trauma, Emergencia y Desastre (CICCATED). Categorización de Centros para la Atención del Paciente Traumatizado en la República Argentina. Bases para la implementación de un Programa Institucional. [Coordinador Acad. Jorge Neira], Buenos Aires: Academia Nacional de Medicina, 2011.
} 
Luego de cumplida la fecha estipulada, la hoja de cálculo generada por la aplicación se descargó y se analizó en MS-Excel.

Las variables categóricas se expresan en porcentaje e intervalo de confianza de $95 \%$, las variables continuas se expresan en mediana y percentiles 25-75. La comparación entre dos muestras se realizó mediante test de Chi cuadrado con corrección de Yates y con test de rangos de Wilcoxon para variables categóricas y numéricas, respectivamente.

El análisis estadístico se llevó a cabo con el programa de código abierto R para Windows versión 3.2.2.

\section{Resultados}

Entre los años 2009 y 2015 se entregaron 7.021 becas a 3.944 integrantes del equipo de salud de ocho hospitales públicos y un sistema prehospitalario de emergencias médicas. En el periodo informado se realizaron 470 cursos, $83 \%$ de los cuales fueron dictados dentro de las instituciones a las que pertenecen los profesionales becados. El 43,5\% de los becados fueron médicos, el $29,6 \%$ enfermeros y el $26,9 \%$ incluyó a otros profesionales y no profesionales. Estas becas corresponden a 1,8 (1-12) beca por persona, con un promedio de 1.000 becas por año (rango 154-1517). El ausentismo registrado en ese período fue de $18,2 \%$.

Según el RC el ausentismo total correspondió a 1.184 personas, de las cuales 756 contaban con una dirección de correo electrónico registrada en la base de datos. De los 756 a los cuales se envió la encuesta, se recibieron 168 encuestas completas respondidas $(22 \%$ de tasa de respuesta).

Del total de encuestas respondidas, $112(66,7 \%)$ correspondieron a mujeres, el rango etario de mayor proporción fue 31-40 años (34,5\%), 51,8\% fueron médicos y 39,2\% fueron enfermeros (Fig. 1).

La proporción en la ocupación de quienes respondieron la encuesta, va en línea con la distribución de las becas de capacitación.

Los servicios con mayor representación en la muestra de respondedores fueron Emergencias y UCI (entre ambos, 52.4\%) Tabla 2.

El 44\% refirió tener relación de dependencia laboral con la institución y el $29,8 \%$ ser becarios o residentes. En este sentido, se interpretó que el 73,8\% pertenecía a la planta permanente de la institución al momento de recibir la beca. Los profesionales que más ausentismo registraron fueron los pertenecientes a la planta (Tabla 3).

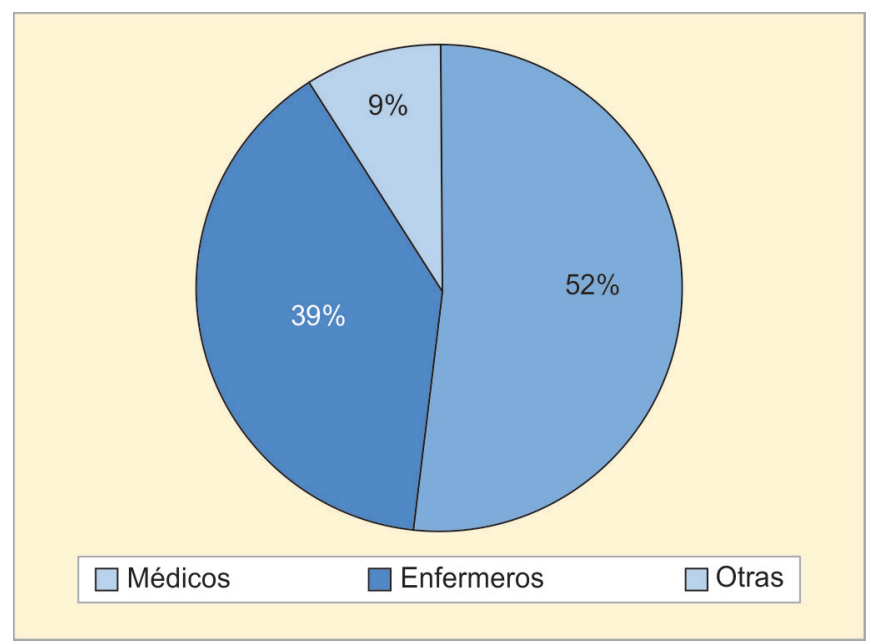

Fig. 1: Ocupación de los usuarios que respondieron la encuesta
Tabla 2: Repondedores por Área o servicio

\begin{tabular}{lcc}
\hline Servicio & Respuestas $(n)$ & Respuestas (\%) \\
\hline Emergencias & 48 & 28,6 \\
UCI/UCIP & 40 & 23,8 \\
Otro & 26 & 15,5 \\
Pediatría & 18 & 10,7 \\
Clínica médica & 16 & 9,5 \\
Cirugía general & 14 & 8,3 \\
Otra & 2 & 1,2 \\
Diagnóstico por imágenes & 2 & 1,2 \\
Ortopedia y traumatología & 2 & 1,2 \\
Total & $\mathbf{1 6 8}$ & $\mathbf{1 0 0 , 0}$ \\
\hline
\end{tabular}

Tabla 3: Ausentismo por rol

\begin{tabular}{lcc}
\hline Rol & Respuestas $(n)$ & Respuestas (\%) \\
\hline Planta & 74 & 44,0 \\
Becario/Residente & 50 & 29,8 \\
Otra & 19 & 11,3 \\
Guardia & 17 & 10,1 \\
Concurrente & 8 & 4,8 \\
Total & $\mathbf{1 6 8}$ & $\mathbf{1 0 0 , 0}$ \\
\hline
\end{tabular}

En la evaluación de los roles profesionales con respecto a las causas de ausentismo, los conflictos laborales fueron los primeros en las causas bien especificadas. Los concurrentes fueron los que tuvieron más conflictos con instituciones fuera de la Red Trauma, mientras que en los profesionales este motivo fue muy bajo, manifestando mayores dificultades dentro de las instituciones de la red.

Con respecto a las causas de ausentismo, el 39,9\% indicó haber tenido dificultades laborales, $14,9 \%$ complicaciones personales, $3 \%$ falta de tiempo para estudiar, 3\% falta de interés en el curso, 2,4\% dificultades para acceder o descargar el material digital de lectura y el 36,9\% no especificó la causa (Tabla 4).

Sólo el 15,5\% manifestó haber estado ausente en más de un curso y un $15,5 \%$ indicó no recordar en qué curso estuvo ausente. $\mathrm{ACLS}^{\circledR}$ fue el curso con el mayor ausentismo según lo relatado por los encuestados (12,5\%), seguido por $\mathrm{CSI}^{\oplus}, \mathrm{ATLS}^{\oplus}$ y $\mathrm{FCCS}^{\oplus}$ (ver Fig. 2).

De los 9 profesionales que manifestaron haber estado ausentes en más de un curso, 4 fueron de planta, 3 becarios o residentes y 2 concurrentes.

\section{Conclusión}

Si bien el Programa de Becas elimina la barrera económica que muchos de los profesionales pueden tener para acceder a la capacitación con respaldo científico, se observa que no es suficiente para garantizar el acceso a los cursos.

Los resultados de la encuesta arrojan información relevante que permite orientar los esfuerzos en diseñar e implementar estrategias para disminuir el ausentismo.

Considerando que los motivos laborales fueron mencionados como la causa principal de ausentismo y que el $83 \%$ de los cursos fue realizado en las instituciones de la Red, se recomienda trabajar con las autoridades sanitarias hospitalarias y ministeriales sobre la identificación de las estrategias específicas para disminuir su impacto, ya sea mediante un sistema de reemplazos, autorizaciones formales, flexibilidad horaria, u otras alternativas que estimulen la 
Tabla 4: Motivo de Ausentismo y Rol

\begin{tabular}{|c|c|c|c|c|c|c|}
\hline Motivo & Planta & Becario/Residente & Guardia & Concurrente & Otra & Total \\
\hline Conflicto con horario laboral en institución de la Red Trauma & 25,7 & 24,0 & 17,6 & 12,5 & 5,3 & 21,4 \\
\hline $\begin{array}{l}\text { Conflicto con horario laboral en institución no perteneciente } \\
\text { a la Red Trauma }\end{array}$ & 8,1 & 24,0 & 35,3 & 50,0 & 15,8 & 18,5 \\
\hline Dificultad personal o familiar & 18,9 & 10,0 & 35,3 & 0,0 & 0,0 & 14,9 \\
\hline El curso no era de su interés & 2,7 & 2,0 & 0,0 & 12,5 & 5,3 & 3,0 \\
\hline Dificultad para estudiar el material & 5,4 & 2,0 & 0,0 & 0,0 & 0,0 & 3,0 \\
\hline Dificultad para acceder o descargar el material de lectura & 4,1 & 2,0 & 0,0 & 0,0 & 0,0 & 2,4 \\
\hline Otro & 35,1 & 36,0 & 11,8 & 25,0 & 73,7 & 36,9 \\
\hline Total & 100,0 & 100,0 & 100,0 & 100,0 & 100,0 & 100,0 \\
\hline
\end{tabular}

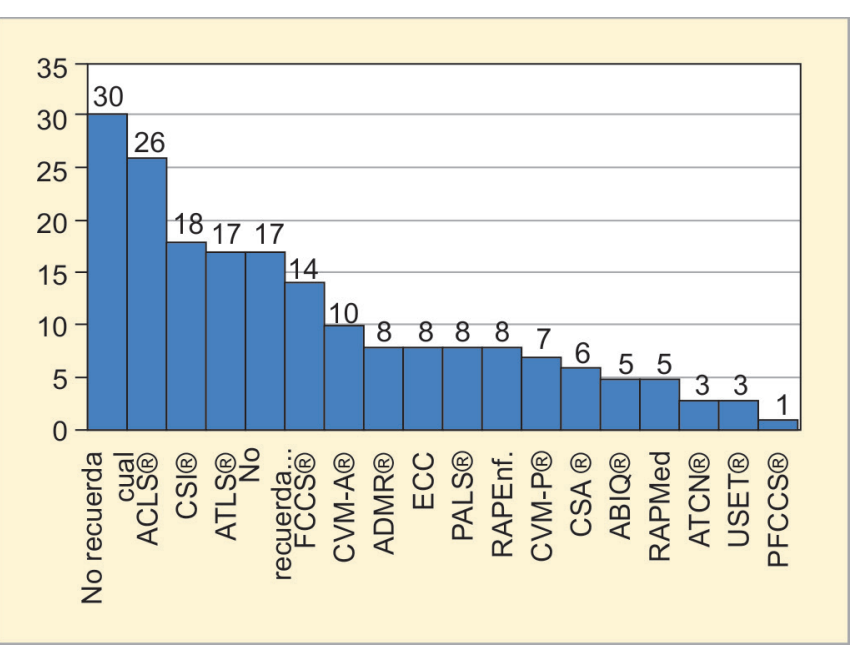

Fig. 2: Cursos en que los encuestados indican no haber asistido

capacitación y la actualización del equipo de salud en cursos con respaldo científico.

Asimismo, se observa que es fundamental fomentar el compromiso de los profesionales que participan del Programa de Becas, y la jerarquización por parte de las autoridades de la formación permanente para aumentar la adhesión a la propuesta.

El objetivo es mejorar el desempeño del rol de los distintos miembros del equipo de salud, con el propósito de contribuir a la mejora de la calidad de atención. En este punto los cursos con respaldo científico, en la medida que ofrecen los protocolos de atención recomendados por las organizaciones rectoras, contribuyen a un mejor desempeño institucional.

\section{Anexo I}

\section{Encuesta de Capacitación | AUSENTISMO}

Estimado/a profesional,

Entre los años 2009-2015 usted fue beneficiado/a con una beca de Fundación Trauma (ex CEDECEM Trauma) para que realizara un curso de capacitación. Según nuestros registros usted no pudo asistir.

Por eso mismo, a continuación le enviamos una encuesta cuyo objetivo es relevar la/s causa/s de ausentismo, con el fin de detectar los principales inconvenientes que afronta el personal de salud a la hora de capacitarse. Es nuestro deseo, que esta valiosa información colabore en el diseño de estrategias que permitan facilitar la capacitación continua sin comprometer las actividades profesionales y personales.
Por esto mismo, les solicitamos que respondan esta encuesta ANÓNIMA con total honestidad.

Por cualquier duda o consulta, no dude en comunicarse con nosotros a capacitacion@fundaciontrauma.org.ar o a (11) 5279-1500.

*Obligatorio

\section{1. ¿Género?* \\ - Femenino \\ - Masculino}

\section{2. ¿Qué edad tenía usted al momento de recibir la beca?*}

- < 21 años

- 21 a 30 años

- 31 a 40 años

- 41 a 50 años

- 51 a 60 años

- De 60 años

\section{3. ¿Cuál es su ocupación? (si posee más de una ocupación, seleccione la que considere principal dentro de la institución)*}

- Médico/a

- Enfermero/a

- Otra

\section{4. ¿A qué institución pertenece/pertenecía usted al momento de recibir la beca?*}

- Hospital El Cruce, F. Varela

- Hospital Erill, Escobar

- Hospital Eurnekian, Ezeiza

- Hospital Güemes, Haedo

- Hospital KM 32, G. Catán

- Hospital San Martín, La Plata

- Hospital San Roque, Gonnet

- Hospital Sor María Ludovica, La Plata

- Otra

\section{5. ¿En qué servicio se desempeña/desempeñaba al momento de recibir la beca? (si posee más de una seleccione la que considere principal)*}

- Emergencias 
- UCI/UCIP

- Clínica Médica

- Pediatría

- Cirugía General

- Diagnóstico por Imágenes

- Ortopedia y Traumatología

- Otro

\section{6. ¿Qué tipo relación laboral tiene/tenía usted con la institución en el momento que recibió la beca?*}

- Becario/Residente

- Concurrente

- Planta

- Guardia

- Otra

\section{7. ¿En qué curso estaba inscrito/a y no asistió?**}

- Atención Básica Inicial del Quemado $\left(\mathrm{ABIQ}^{\circledR}\right)$

- Advanced Cardiac Life Support (ACLS $\left.{ }^{\oplus}\right)$

- Advanced Disaster Medical Response (ADMR $\left.{ }^{\circledR}\right)$

- Advanced Trauma Life Support (ATLS $\left.{ }^{\circledast}\right)$

- Advanced Trauma Care for Nurses $\left(\right.$ ATCN $\left.^{\circledR}\right)$

- Curso de Socorrismo Intermedio (CSI $\left.{ }^{\circledR}\right)$

- Curso de Socorrismo Avanzado (CSA $\left.{ }^{\oplus}\right)$

- Curso de Ventilación Mecánica en Adultos (CVM-A $\left.{ }^{\oplus}\right)$

- Curso de Ventilación Mecánica Pediátrica (CVM-P®)

- Enfermería en Cuidados Críticos (ECC $\left.{ }^{\circledast}\right)$
- Fundamental Critical Care Support (FCCS $\left.{ }^{\circledR}\right)$

- Pediatric Advanced Life Support (PALS $\left.{ }^{\circledR}\right)$

- Pediatric Fundamental Critical Care Support (PFCCS ${ }^{\circledR}$ )

- Reanimación Avanzada Pediátrica para Médicos (RAPMéd.)

- Reanimación Avanzada Pediátrica para Enfermeros (RAPEnf.)

- Ultrasonografía en Emergencias y Trauma (USET ${ }^{\oplus}$ )

- Estuvo inscrito/a a un curso, pero no recuerda cuál

- No recuerda haber estado inscrito/a en un curso

\section{8. ¿Por qué motivo no asistió al curso?*}

- El curso no era de su interés

- No pudo cubrir una guardia/horario laboral para asistir dentro de la institución de la Red Trauma

- No pudo cubrir una guardia/horario laboral para asistir en otra institución no perteneciente a la Red Trauma

- No tuvo tiempo suficiente para estudiar el material

- Tuvo dificultades para acceder o descargar el material de lectura

- No pudo organizarse personalmente o con su familia para asistir

- Otro

\section{¿Tuvo inasistencia a otro curso?*}

- Sí

- No

En el caso que el profesional haya estado ausente en más de un curso, las preguntas 7 y 8 se repiten hasta dos veces más, llegando a las 12 preguntas totales. 